\title{
Risk of Endometriosis and Subsequent Ovary and Breast Cancers in Nurses: A Population-Based Cohort Study in Taiwan
}

\author{
Hsing-Chi Hsu ${ }^{1}$, Kai-Yu Tseng ${ }^{2}$, Hsiang-Chi Wang ${ }^{3,4}$, Fung-Chan Sung ${ }^{3,5,+}$ and \\ Wei-Fen Ma $6,7, t, *$ (i) \\ 1 Department of Public Health, China Medical University College of Public Health, Taichung 404, Taiwan; \\ chiqueens@gmail.com \\ 2 School of Nursing, Central Taiwan University of Science and Technology, Taichung 404, Taiwan; \\ 107179@ctust.edu.tw \\ 3 Management Office for Health Data, China Medical University Hospital, Taichung 404, Taiwan; \\ vwang.lk@gmail.com (H.-C.W.); fcsung1008@yahoo.com (F.-C.S.) \\ 4 College of Medicine, China Medical University, Taichung 404, Taiwan \\ 5 Department of Health Services Administration, China Medical University College of Public Health, \\ Taichung 404, Taiwan \\ 6 School of Nursing, China Medical University, Taichung 404, Taiwan \\ 7 Department of Nursing, China Medical University Hospital, Taichung 404, Taiwan \\ * Correspondence: 1hdaisy@mail.cmu.edu.tw; Tel.: +886-4-2205-3366 (ext. 7107); Fax: +886-4-2205-3748 \\ + These authors contributed equally to the research.
}

Received: 15 August 2019; Accepted: 13 September 2019; Published: 18 September 2019

\begin{abstract}
Background: Endometriosis has been associated with the subsequent development of ovarian and breast cancers. This study evaluated whether nurses were at increased risks of developing endometriosis and subsequent ovarian and breast cancers. Methods: From Taiwan National Health Insurance Research Database during 2000 to 2011, we established 3 study cohorts, consisting of 23,801 nurses, 11,973 other hospital employees, and 143,096 general women free of endometriosis and cancer. Women in all cohorts were followed to the end of 2011 to measure the occurrences of endometriosis and subsequent ovarian and breast cancers. The incident endometriosis cases and related hazard ratio (HR) and 95\% confidence interval (CI) were calculated. The incident cases of ovarian cancer and breast cancer and related odds ratio were calculated. Results: The incidence of endometriosis was the highest in the nurse cohort (4.23 per 100, $n=966)$ followed by other health professionals (3.74 per 100, $n=427$ ) and control cohort (3.06 per 100, $n=4193)$, with adjusted hazard ratios of $1.28(95 \% \mathrm{CI}=1.20-1.38)$ and $1.13(95 \% \mathrm{CI}=1.02-1.25)$, respectively, comparing to controls. Among those who developed endometriosis, nurses had higher subsequent ovarian cancer and lower breast cancer, but not significant. Conclusions: Nurses are at a higher risk of developing endometriosis. However, the link between endometriosis and subsequent cancers is weak.
\end{abstract}

Keywords: endometriosis; breast cancer; nurses; ovarian cancer; retrospective cohort study

\section{Introduction}

A systematic review showed that endometriosis is one of the most common gynecological diseases among women of childbearing age, with the prevalence rates range $2-10 \%$ among countries $[1,2]$. Causes of endometriosis have been associated with menstrual reflux, hormonal imbalance, heredity, specific genes, and environment and immune anomaly, but remain unclear [2-4]. The main clinical symptoms of endometriosis include abnormal bleeding, dysmenorrhea, chronic pelvic pain, and 
infertility $[1,2,5]$. Up to $50 \%$ of endometriosis women of childbearing age suffer from infertility [6], and these symptoms lead to negative psychological effects [7].

Women with endometriosis are at an increased risk of developing other complications. Previous studies have found that women with endometriosis are at an increased risk of epithelial ovarian cancer, with hazard ratios (HRs) of epithelial ovarian cancer range from 2.01 to 4.56 [8-10].

Clinical nursing is a high-stress work. Nurses need to deal with emergency cares for patients with the challenge of lifestyle change. Approximately, 85\% nurses in Taiwan have reported that working stress is the major risk factor to threaten their health [11]. Marino et al. have indicated that health professionals, particularly nurses, were at increased risks of developing endometriosis, with an adjusted odds ratio $(\mathrm{OR})$ of $1.60(95 \% \mathrm{CI}=1.00-2.55)$ [12]. In addition, a recent study in Taiwan found that the nurses have increased risks than general female population for breast cancer (standardized incidence ratio $(\mathrm{SIR})=1.2$ ) and ovarian cancer (SIR = 1.8) [13]. An American Nurse Health study also found nurses with endometriosis are at an increased risk of cancer with a relative risk of 1.81 for ovarian cancer [14]. Another study in Taiwan also found that the incidence of cancer in nurses with endometriosis increases with age [13].

Women with endometriosis are characterized with elevated estrogen and subsequently are at increased risk of breast cancer, ovarian cancer, and benign breast diseases $[15,16]$. Whether the presence of endometriosis aggravating the risk of ovarian and breast cancers in nurses has not been well investigated among populations. We, therefore, attempted to use the insurance claims data of Taiwan to evaluate the risk of developing endometriosis and subsequent risk of developing ovarian and breast cancers, comparing among nurses, other healthcare professionals, and non-healthcare professionals.

\section{Materials and Methods}

\subsection{Data Sources}

The present study used the National Health Insurance Research Database (NHIRD) of Taiwan from 1996 to 2011. Demographic status of insured population, treatment and medications received, and cost of care are available in the database. Diseases were coded with the International Classification of Diseases, Ninth Revision, Clinical Modification (ICD-9-CM). A subset Registry for Medical Personnel database containing the information of healthcare professionals was used to identify nurses and other types of healthcare professionals. The said information was gathered from the Registry for Contracted Medical Facilities, Registry for Prescription Files, and Medical Claims Data in the NHIRD. The personal information included identification, sex, birth date, employer, and dates of employment initiation and termination. To protect personal privacy, all identifications in the NHIRD data were encrypted by the National Health Research Institutes. The study was approved by the Research Ethics Committee, China Medical University and Hospital (IRB permits number: CMUH-104-REC2-115).

\subsection{Study Population}

From the Registry for Medical Personnel database, we identified 23,810 nurses aged 20-54 years without history of endometriosis and cancer. From 55,323 females of other healthcare professionals (physicians, dentists, pharmacists, dietitians, radiologists, and physical therapists), we randomly selected 11,973 women as "other hospital employees" cohort, frequency matched with the nurse cohort by age, without the history of endometriosis and cancer. We further randomly selected 143,096 women without history of endometriosis or cancer from general female population frequency matched with the nurse cohort and other professional cohorts by age as the control cohort.

\subsection{Study Outcome and Comorbidity}

The primary outcome of the study was endometriosis defined by ICD-9-CM (code 617), newly diagnosed in outpatients or inpatients data after the baseline date. Women in the 3 cohorts were followed up to the date of endometriosis diagnosis, withdrawal from insurance, or the end of 
31 December, 2011. The study further followed women who had developed endometriosis for the occurrence of ovarian and breast cancers as secondary outcomes. Claim records of pelvic inflammatory disease (PID) (ICD-9-CM code 614), cardiovascular disease (CVD) (ICD-9-CM code 410-414, 428, 430-438, 440-448), infertility (ICD-9-CM code 606, 628), diabetes mellitus (ICD-9-CM code 250), chronic liver disease (ICD-9-CM code 275.0), leiomyoma of uterus (ICD-9-CM code 218), and autoimmune disease (ICD-9-CM code 710.0, 714.0, 340) were identified at the baseline as comorbidities.

\subsection{Statistical Analysis}

Baseline characteristics presented as categorical variables were tested with $\chi^{2}$ test. ANOVA test was used to examine means among cohorts. Incident endometriosis cases were estimated for the 3 cohorts and the Cox proportional hazards regression analysis was applied to estimate the hazard ratio (HR) of endometriosis and related 95\% confidence interval (CI). The adjusted HR (aHR) was estimated using multivariable regression analysis by including study cohort, age, and comorbidities with significant crude HR (cHR). Subsequent cases of ovarian cancer and breast cancer were estimated for women who had developed endometriosis among the 3 study cohorts. We used logistic regression analysis to calculate the odds ratio (OR) of developing ovarian cancer and breast cancer among women with endometriosis by study cohort and age. We used SAS statistical analysis software, Version 9.4 (SAS Institute Inc., Cary, North Carolina) to conduct data analyses with the significance level of 0.05.

\section{Results}

The study population had a mean age of 34 years, and approximately $42 \%$ of women aged $30-39$ years (Table 1). Baseline comorbidities were more prevalent in the control cohort than in other two cohorts, particularly of cardiovascular disease and diabetes mellitus.

Table 2 shows that the occurrence of endometriosis was the highest in the nurse cohort (4.06 per $100, n=966)$, followed by the other hospital employees ( 3.57 per 100, $n=427)$ and the control cohort (2.93 per 100, $n=4193)$. The aHRs of endometriosis were 1.28 (95\% CI, $1.20-1.38)(p<0.0001)$ for the nurse cohort and $1.13(95 \%$ CI, 1.02-1.25) $(p=0.018)$ for other hospital employees, compared to the control cohort. Women aged 30-39 years had the highest aHR of endometriosis among age groups, compared with the youngest group. Women with pelvic inflammatory disease and infertility were also at a higher hazard of developing endometriosis.

Table 3 shows that 10 ovarian cancer cases and 37 breast cancer cases subsequently occurred among women who had developed endometriosis during the follow-up period. The nurse cohort had a higher incidence of ovarian cancer and a lower incidence of breast cancer than other 2 cohorts, but not significant, compared with the control cohort. Other hospital employees had higher incident ovarian cancer and breast cancer than the control cohort had, but not significant. There was no significant variation in ovarian cancer cases among age groups. The estimated breast cancer risk increased with age, with an adjusted OR of $14.8(95 \% \mathrm{CI}=1.90-98.7)$ for women aged $40-49$ years. 
Table 1. Demographic characteristics and comorbidities.

\begin{tabular}{|c|c|c|c|c|c|c|c|}
\hline \multirow{4}{*}{ Variable } & \multicolumn{6}{|c|}{ Study Cohorts } & \multirow{4}{*}{$p$-Value } \\
\hline & \multirow{2}{*}{\multicolumn{2}{|c|}{$\begin{array}{c}\text { Controls } \\
n=143,096\end{array}$}} & \multirow{2}{*}{\multicolumn{2}{|c|}{$\begin{array}{c}\text { Nurses } \\
n=23,801\end{array}$}} & \multirow{2}{*}{\multicolumn{2}{|c|}{$\begin{array}{c}\text { Other Hospital Employee * } \\
\qquad n=11,973\end{array}$}} & \\
\hline & & & & & & & \\
\hline & $n$ & $\%$ & $n$ & $\%$ & $n$ & $\%$ & \\
\hline \multicolumn{8}{|l|}{ Age, Years ${ }^{\dagger}$} \\
\hline $20-29$ & 48,816 & 34.1 & 8136 & 34.2 & 4068 & 34.0 & 0.35 \\
\hline $30-39$ & 60,564 & 42.3 & 10,094 & 42.4 & 5047 & 42.2 & \\
\hline $40-49$ & 27,588 & 19.3 & 4598 & 19.3 & 2299 & 19.2 & \\
\hline $50-54$ & 3504 & 2.45 & 584 & 2.45 & 292 & 2.44 & \\
\hline Unknown & 2624 & 1.83 & 389 & 1.63 & 267 & 2.23 & \\
\hline Mean (SD) & \multicolumn{2}{|c|}{$34.0(7.74)$} & \multicolumn{2}{|c|}{$34.0(7.66)$} & \multicolumn{2}{|c|}{$34.1(7.52)$} & 0.25 \\
\hline \multicolumn{8}{|l|}{ Comorbidities } \\
\hline PID & 981 & 0.69 & 145 & 0.61 & 52 & 0.43 & 0.003 \\
\hline Infertility & 98 & 0.07 & 16 & 0.07 & 10 & 0.08 & 0.83 \\
\hline CVD & 646 & 0.45 & 59 & 0.25 & 34 & 0.28 & $<0.0001$ \\
\hline $\mathrm{DM}$ & 584 & 0.41 & 32 & 0.13 & 20 & 0.17 & $<0.0001$ \\
\hline Leiomyoma of uterus & 782 & 0.55 & 133 & 0.56 & 49 & 0.41 & 0.13 \\
\hline Autoimmune & 109 & 0.08 & 26 & 0.11 & 7 & 0.06 & 0.17 \\
\hline
\end{tabular}

* Physician, pharmacist, dentist, dietitian, radiologist, physiotherapist. $p$-value: Chi-square test for categorical variable, ANOVA for means. ${ }^{\dagger} 3280$ women with missing age. Abbreviation: SD: standard deviation; PID: Pelvic inflammatory disease, CVD: Cardiovascular disease, DM: diabetes mellitus. 
Table 2. Endometriosis cases by study cohort, age, and comorbidity and Cox method estimated hazard ratios of endometriosis

\begin{tabular}{|c|c|c|c|c|c|}
\hline \multirow{2}{*}{ Variable } & \multirow{2}{*}{$\begin{array}{c}\text { Endometriosis } \\
(n=5586)\end{array}$} & \multicolumn{2}{|c|}{ Crude } & \multicolumn{2}{|c|}{ Adjusted $^{\dagger}$} \\
\hline & & HR $(95 \%$ CI) & $p$-Value & HR $(95 \%$ CI) & $p$-Value \\
\hline Occupation & $n$ & & & & \\
\hline Controls & 4193 & 1 (Reference) & & 1 (Reference) & \\
\hline Nurses & 966 & $1.28(1.19,1.37)$ & $<0.0001$ & $1.28(1.20,1.38)$ & $<0.0001$ \\
\hline Other hospital employees * & 427 & $1.12(1.01,1.24)$ & 0.027 & $1.13(1.02,1.25)$ & 0.018 \\
\hline \multicolumn{6}{|l|}{ Age, years } \\
\hline $20-29$ & 1332 & 1 (Reference) & & 1 (Reference) & \\
\hline $30-39$ & 3186 & $1.81(1.69,1.92)$ & $<0.0001$ & $1.81(1.70,1.93)$ & $<0.0001$ \\
\hline $40-49$ & 1037 & $1.24(1.15,1.35)$ & $<0.0001$ & $1.25(1.15,1.35)$ & $<0.0001$ \\
\hline $50-54$ & 24 & $0.22(0.15,0.34)$ & $<0.0001$ & $0.23(0.15,0.34)$ & $<0.0001$ \\
\hline \multicolumn{6}{|l|}{ Comorbidities } \\
\hline PID & 61 & $1.58(1.23,2.03)$ & 0.0004 & $1.41(1.09,1.83)$ & 0.01 \\
\hline Infertility & 11 & $2.70(1.49,4.87)$ & 0.001 & $1.97(1.07,3.62)$ & 0.03 \\
\hline CVD & 20 & $0.95(0.61,1.48)$ & 0.82 & & \\
\hline $\mathrm{DM}$ & 9 & $0.50(0.26,0.96)$ & 0.04 & & \\
\hline Leiomyoma of uterus & 36 & $1.12(0.81,1.56)$ & 0.49 & & \\
\hline Autoimmune disease & 6 & $1.40(0.63,3.11)$ & 0.41 & & \\
\hline
\end{tabular}

*: Physician, pharmacist, dentist, dietitian, radiologist, physiotherapist. HR, Cox regression model estimated hazard ratio; Adjusted HR ${ }^{\dagger}$ estimated using multivariable analysis; $\mathrm{CI}_{\text {, }}$ confidence interval. SD, standard deviation; PID, Pelvic inflammatory disease; CVD, Cardiovascular disease; DM, diabetes mellitus. 
Table 3. Cases of ovary cancer and breast cancer developed among women with endometriosis and odds ratio of developing cancer by study cohort and age.

\begin{tabular}{|c|c|c|c|c|c|c|c|c|c|c|c|c|c|c|c|c|}
\hline \multirow{3}{*}{ Variables } & \multicolumn{10}{|c|}{ Ovary Cancer } & \multicolumn{6}{|c|}{ Breast Cancer } \\
\hline & \multicolumn{2}{|c|}{ Cases } & \multicolumn{3}{|c|}{ Crude } & \multicolumn{3}{|c|}{ Adjusted } & \multicolumn{2}{|c|}{ Cases } & \multicolumn{3}{|c|}{ Crude } & \multicolumn{3}{|c|}{ Adjusted } \\
\hline & Yes & No & OR & $95 \% \mathrm{CI}$ & $p$ & OR & $95 \% \mathrm{CI}$ & $p$ & Yes & No & OR & $95 \% \mathrm{CI}$ & $p$ & OR & $95 \% \mathrm{CI}$ & $p$ \\
\hline \multicolumn{17}{|l|}{ Study cohort } \\
\hline Controls & 6 & 4187 & 1 & Reference & & 1 & Reference & & 28 & 4165 & 1 & Reference & & 1 & Reference & \\
\hline Nurses & 3 & 963 & 2.18 & $(0.54,8.73)$ & 0.27 & 2.13 & $(0.53,8.53)$ & 0.27 & 4 & 962 & 0.62 & $(0.22,1.77)$ & 0.37 & 0.62 & $(0.22,1.78)$ & 0.38 \\
\hline Other hospital employees * & 1 & 426 & 1.64 & $(0.20,13.7)$ & 0.65 & 1.60 & $(0.19,13.4)$ & 0.67 & 5 & 422 & 1.77 & $(0.68,4.60)$ & 0.24 & 1.97 & $(0.75,5.13)$ & 0.17 \\
\hline \multicolumn{17}{|l|}{ Age, years } \\
\hline $20-29$ & 3 & 1329 & 1 & Reference & & 1 & Reference & & 1 & 1331 & 1 & Reference & & 1 & Reference & \\
\hline $30-39$ & 4 & 3182 & 0.56 & $(0.12,2.49)$ & 0.44 & 0.57 & $(0.13,2.56)$ & 0.46 & 25 & 3161 & 10.5 & $(1.42,77.8)$ & 0.02 & 10.8 & $(1.46,80.0)$ & 0.02 \\
\hline $40-49$ & 3 & 1034 & 1.29 & $(0.26,6.38)$ & 0.76 & 1.29 & $(0.26,6.43)$ & 0.75 & 11 & 1026 & 14.3 & $(1.84,99.8)$ & 0.01 & 14.8 & $(1.90,98.7)$ & 0.01 \\
\hline $50-55$ & 0 & 24 & 0 & & & & & & 0 & 24 & - & & & - & & \\
\hline
\end{tabular}




\section{Discussion}

Our study results showed that among the 3 study cohorts, the nurse cohort had an incidence of endometriosis $14 \%$ higher than the other female health professionals or $38 \%$ higher than the control cohort, with an aHR of 1.28 relative to the control cohort. Other female health professionals also had the incidence of endometriosis significantly higher than the control cohort. The data indicate that health professionals are more likely to be diagnosed for endometriosis.

Previous studies have demonstrated that hyperlipidemia and hypertension [17], obesity, diabetes $[8,18,19]$, and comorbidities of PID, infertility, CVD, chronic liver disease, and rheumatic diseases are prevalent in women with endometriosis $[17,18]$. We compared the baseline comorbidities among the three study cohorts and found that PID, CVD, and diabetes mellitus (DM) were the main comorbidities which were greater in the control cohort. It is interesting to note that PID and infertility were marked as only significant comorbidities associated with developing endometriosis in the present study (Table 2). The relationship between PID and endometriosis could be stronger for the nurse cohort and the other health professionals than the control cohort. The endometriosis risk is thus greater in the nurse cohort and other health professionals than in the control cohort. Nurses are more likely to change jobs than other health employees. Moreover, a large portion of nurses has a night-shift work style. Marino et al. also found that nurses or health aides are at particular higher risk of endometriosis [12]. They also found that working tenure and education did not have an association with an increased risk of endometriosis.

The age-specific data in this study showed that women aged 30-39 years were at the highest hazard to develop endometriosis than women aged 40-49 years, compared with the 20-29 years old group. The risk of endometriosis plummeted to $77 \%$ in women beyond 49 years. A German study evaluated 20,835 hospitalized women with endometriosis and found that $39.7 \%$ of cases were 30-39 years old or $78.4 \%$ of cases were $20-44$ years old [19]. The percentage reduced to $7.1 \%$ in women aged 50 years and older, which may be associated with a decline in ovarian function leading to the atrophy of the endometrium and to the reduction of endometriosis symptoms [1]. They also noted that nurses with endometriosis were more likely to have the comorbidities of PID and infertility than nurses without endometriosis [20]. Our study demonstrated a similar relationship. It is well known that PID and endometriosis share similar symptom of pelvic pain. Some PID cases may actually a misdiagnosis of endometriosis made earlier in the patient's life. However, it is important to note that the mean age from the onset of PID symptoms to the diagnosis of endometriosis was 10.4 years [21]. Early diagnosis of endometriosis may be delayed due to the confused or similar symptoms of PID. Therefore, early and accurate diagnosis is more crucial.

Previous studies have confirmed a correlation between endometriosis with ovarian cancer [8-10,14]. Our study failed to show significant difference among the 3 study cohorts in the relationships between endometriosis and ovarian cancer and between endometriosis and breast cancer. This is because of small number of ovarian cancer and breast cancer cases. The follow-up time is likely not long enough for women with endometriosis to develop these two cancers. However, our study did show that the breast cancer risk increased with age when all women with endometriosis in the 3 cohorts were pooled for the data analysis. Previous studies with a large general female population found women with endometriosis are at an increased risk of developing breast cancer after a long follow-up time $[9,15]$. Anifantaki et al. concluded in a review that the risk of breast cancer increases with age for women with endometriosis [22].

\section{Limitations}

This study is a population-based data to evaluate the risk of developing endometriosis and subsequent ovarian cancer and breast cancer among nurses, other healthcare employees, and general women. However, information on lifestyle, social activities, job tenure, and the level of stress at work in the study population was unavailable. We were unable to include these factors in the data analysis. Further study needs to include these factors with a larger cohort size and longer follow-up period. 


\section{Conclusions}

This study demonstrated that nurses and other health workers are at a higher risk of developing endometriosis than are general women. PID and infertility could be also associated with the development of endometriosis. Being a nurse or other health worker should be a protective factor instead of an occupational risk. However, whether nurses at a higher risk for endometriosis because of stress needs investigation with more studies. Further study also requires a larger sample with a longer follow-up period to evaluate subsequent cancer risk among those with endometriosis.

Author Contributions: H.-C.H., K.-Y.T., and W.-F.M. initiated the study idea; all authors designed the study; H.-C.H., H.-C.W., and F.-C.S. analyzed and interpreted data; all authors drafted the manuscript; K.-Y.T. and F.-C.S. revised the manuscript. W.-F.M. supervised the study. All authors read and approved the final manuscript.

Funding: This work was funded by grants from the Ministry of Health and Welfare, Taiwan (MOHW107-TDU-B-212-123004), China Medical University (CMU108-MF-34), China Medical University Hospital, Academia Sinica Stroke Biosignature Project (BM10701010021), Ministry of Science and Technology(MOST108-2314-B-039-004), MOST Clinical Trial Consortium for Stroke (MOST106-2321-B-039-005), Tseng-Lien Lin Foundation, Taichung, Taiwan, and Katsuzo and Kiyo Aoshima Memorial Funds, Japan. The funders had no role in the study design, data collection and analysis, publication decision, or manuscript drafting.

Conflicts of Interest: The authors declare that they have no competing interests involved.

\section{Abbreviations}

\begin{tabular}{|c|c|}
\hline aHR & adjusted hazard ratio; \\
\hline $\mathrm{cHR}$ & crude adjusted hazard ratio; \\
\hline $\mathrm{CI}$ & confidence interval; \\
\hline CVD & cardiovascular disease; \\
\hline HR & hazard ratios; \\
\hline ICD-9-CM & $\begin{array}{l}\text { International Classification of Diseases Ninth } \\
\text { Revision Clinical Modification; }\end{array}$ \\
\hline NHIRD & National Health Insurance Research Database; \\
\hline OR & odds ratio; \\
\hline PID & pelvic inflammatory disease; \\
\hline SIR & standardized incidence ratio. \\
\hline
\end{tabular}

\section{References}

1. Giudice, L.C.; Kao, L.C. Endometriosis. Lancet 2004, 364, 1789-1799. [CrossRef]

2. Hickey, M.; Ballard, K.; Farquhar, C. Endometriosis. Br. Med. J. 2014, 348, g1752. [CrossRef] [PubMed]

3. Teng, S.-W.; Horng, H.-C.; Ho, C.-H.; Yen, M.-S.; Chao, H.-T.; Wang, P.-H.; Chuang, C.M. Women with endometriosis have higher comorbidities: Analysis of domestic data in Taiwan. J. Chin. Med. Assoc. 2016, 79, 577-582. [CrossRef] [PubMed]

4. Vinatier, D.; Orazi, G.; Cosson, M.; Dufour, P. Theories of endometriosis. Eur. J. Obstet. Gynecol. Reprod. Biol. 2001, 96, 21-34. [CrossRef]

5. Nnoaham, K.E.; Hummelshoj, L.; Webster, P.; d'Hooghe, T.; de Cicco Nardone, F.; de Cicco Nardone, C.; World Endometriosis Research Foundation Global Study. Impact of endometriosis on quality of life and work productivity: A multicenter study across ten countries. Fertil. Steril. 2011, 96, 366-373. [CrossRef]

6. Meuleman, C.; Vandenabeele, B.; Fieuws, S.; Spiessens, C.; Timmerman, D.; D'Hooghe, T. High prevalence of endometriosis in infertile women with normal ovulation and normospermic partners. Fertil. Steril. 2009, 92, 68-74. [CrossRef] [PubMed]

7. De Graaff, A.A.; D’hooghe, T.M.; Dunselman, G.A.J.; Dirksen, C.D.; Hummelshoj, L.; WERF EndoCost Consortium; Canis, M. The significant effect of endometriosis on physical, mental and social wellbeing: Results from an international cross-sectional survey. Hum. Reprod. 2013, 28, 2677-2685. [CrossRef] [PubMed]

8. Chang, W.-H.; Wang, K.-C.; Lee, W.-L.; Huang, N.; Chou, Y.-J.; Feng, R.-C.; Yen, M.-S.; Huang, B.-S.; Gou, C.-Y.; Wang, P.-H. Endometriosis and the subsequent risk of epithelial ovarian cancer. Taiwan J. Obstet. Gynecol. 2014, 53, 530-535. [CrossRef] [PubMed] 
9. Kok, V.C.; Tsai, H.-J.; Su, C.-F.; Lee, C.-K. The Risks for Ovarian, Endometrial, Breast, Colorectal, and Other Cancers in Women with Newly Diagnosed Endometriosis or Adenomyosis: A Population-Based Study. Int. J. Gynecol. Cancer 2015, 25, 968-976. [CrossRef] [PubMed]

10. Lee, W.-L.; Chang, W.-H.; Wang, K.-C.; Guo, C.-Y.; Chou, Y.-J.; Huang, N.; Huang, N.; Yen, M.-S.; Wang, P.-H. The Risk of Epithelial Ovarian Cancer of Women with Endometriosis May be Varied Greatly if Diagnostic Criteria Are Different: A Nationwide Population-Based Cohort Study. Medicine 2015, 94, e1633. [CrossRef] [PubMed]

11. Lee, S.; Pai, H.C.; Yen, W.J. A study of perceived practice environment among nurses in Taiwan. Hu Li Za Zhi 2008, 55, 30-40. [PubMed]

12. Marino, J.L.; Holt, V.L.; Chen, C.; Davis, S. Lifetime occupational history and risk of endometriosis. Scand. J. Work Environ. Health 2009, 35, 233-240. [CrossRef] [PubMed]

13. Hou, Y.-J. Incidence Risk of Cancers and Situational Analysis among Nurses during the 2008-2012 Years. Master's Thesis, Department of Nursing, Kaohsiung Medical University, Kaohsiung City, Taiwan, 2016. Available online: http://ir.kmu.edu.tw/handle/310902000/40239 (accessed on 15 November 2018).

14. Poole, E.M.; Lin, W.T.; Kvaskoff, M.; De Vivo, I.; Terry, K.L.; Missmer, S.A. Endometriosis and risk of ovarian and endometrial cancers in a large prospective cohort of U.S. nurses. Cancer Causes Control 2017, 28, 437-445. [CrossRef] [PubMed]

15. Chuang, S.-C.; Wu, G.-J.; Lu, Y.-S.; Lin, C.-H.; Hsiung, C.A. Associations between Medical Conditions and Breast Cancer Risk in Asians: A Nationwide Population-Based Study in Taiwan. PLoS ONE 2015, 10, e0143410. [CrossRef] [PubMed]

16. Farland, L.V.; Tamimi, R.M.; Heather Eliassen, A.; Spiegelman, D.; Collins, L.C.; Schnitt, S.J.; Missmer, S.A. A prospective study of endometriosis and risk of benign breast disease. Breast Cancer Res. Treat. 2016, 159, 545-552. [CrossRef] [PubMed]

17. Mu, F.; Rich-Edwards, J.; Rimm, E.B.; Spiegelman, D.; Missmer, S.A. Endometriosis and Risk of Coronary Heart Disease. Circ. Cardiovasc. Qual. Outcomes 2016, 9, 257-264. [CrossRef] [PubMed]

18. Wang, K.-C.; Chang, W.-H.; Lee, W.-L.; Huang, N.; Huang, H.-Y.; Yen, M.-S.; Wang, P.H. An increased risk of epithelial ovarian cancer in Taiwanese women with a new surgico-pathological diagnosis of endometriosis. BMC Cancer 2014, 14, 831. [CrossRef] [PubMed]

19. Yu, H.-C.; Lin, C.-Y.; Chang, W.-C.; Shen, B.-J.; Chang, W.-P.; Chuang, C.-M. Increased Association Between Endometriosis and Endometrial Cancer: A Nationwide Population-Based Retrospective Cohort Study. Int. J. Gynecol. Cancer 2015, 25, 447-452. [CrossRef]

20. Haas, D.; Chvatal, R.; Reichert, B.; Renner, S.; Shebl, O.; Binder, H.; Oppelt, P. Endometriosis: A premenopausal disease? Age pattern in 42,079 patients with endometriosis. Arch. Gynecol. Obstet. 2012, 286, 667-670. [CrossRef]

21. Hudelist, G.; Fritzer, N.; Thomas, A.; Niehues, C.; Oppelt, P.; Haas, D.; Tammaa, A.; Salzer, H. Diagnostic delay for endometriosis in Austria and Germany: Causes and possible consequences. Hum. Reprod. 2012, 27, 3412-3416. [CrossRef]

22. Anifantaki, F.; Boutas, I.; Kalampokas, T.; Kalampokas, E.; Sofoudis, C.; Salakos, N. Association of endometriosis and breast cancer: Mini review of the literature. Arch. Gynecol. Obstet. 2016, 293, 5-10. [CrossRef] [PubMed]

(C) 2019 by the authors. Licensee MDPI, Basel, Switzerland. This article is an open access article distributed under the terms and conditions of the Creative Commons Attribution (CC BY) license (http://creativecommons.org/licenses/by/4.0/). 